\title{
MAS ALLÁ DE LA CLASIFICACIÓN Y LA COMPRENSIÓN DE LOS CONCEPTOS DE LOS GRUPOS DE DISCAPACIDAD: LA PERSONA
}

\author{
Ronald Soto Calderón
}

\begin{abstract}
Resumen: Este trabajo presenta aspectos básicos sobre los conceptos de los grupos en los cuales ban sido clasificadas las limitaciones, para posteriormente definir otros conceptos relacionados con los nuevos enfoques sobre discapacidad, en la segunda parte del articulo, se presentan una serie de ideas relacionadas con la forma en que la sociedad debe desarrollar estrategias para favorecer la igualdad y equiparación de oportundiades para las personas con discapacidad.
\end{abstract}

\section{Introducción}

Conscientes de la importancia de un adecuado uso y utilización de los conceptos que sobre discapacidad se han desarrollado, se ha considerado necesario referirse a ellos en la primera parte de este artículo.

Estos conceptos pueden ser muy conocidos, sin embargo, esperamos que los lectores se enriquezcan en la forma en que deben ser analizados e interpretados, de tal manera que la referencia en cuanto a las personas con alguna condición discapacitante sea tratada con el mayor respeto y siempre dentro de una perspectiva ecológica, en la que todo lo relacionado con la discapacidad se centra en la persona y su entorno. Al respecto, comentan Walker y Shea, 1987 "el impacto, actual y potencial del ambiente en la conducta humana es un tema dominante en la sociedad contemporánea" (p. 9). Por lo tanto, se debe considerar importante la interacción que se da entre la persona y su entorno, y determinar de qué manera el entorno influye en el desarrollo integral de la persona, y de qué manera el entorno favorece u obstruye el acceso de la persona con discapacidad a todas aquellas actividades que le corresponde realizar como "tarea de desarrollo" según Erickson, comentado por Papalia y Wendkos (1998) y Hurlock, 1990, de tal manera que al participar la persona en igualdad de condiciones e igualdad de oportunidad, logre una mejor calidad de vida y una mayor participación 
en el entorno en que se desarrolla; y no en un modelo médico (diagnóstico) únicamente, como se ha manejado hasta el momento.

El modelo médico considera que las condiciones discapacitantes deben ser tratadas como síntomas de enfermedades orgánicas para las que se debe encontrar una etiología orgánica, Walker y Shea (1987) comentan que "los defectos orgánicos pueden ser una consecuencia de la herencia o del ambiente (trauma). Los efectos del ambiente pueden ocurrir antes, durante o después del nacimiento." ( $p .8$ ), el modelo considera que una vez sean corregidas las causas de los síntomas ellos desaparecerán, o sea, todas aquellas alteraciones que se provocan en el actuar de las personas con discapacidad, no se presentarán más en las personas por la intervención médica que ha sido practicada.

Es importante aclarar que este modelo médico se ha mostrado muy eficaz en el campo de las enfermedades físicas, y es ahí donde tiene su área de aplicación, sin embargo, esta situación ha hecho que los profesionales del área médica extiendan el modelo, sin la mayor evidencia, sin considerar que el hecho de que no se conozca la causa subyacente no permite concluir que no exista, pues lo único que confirma esta situación, es que el conocimiento actual no ha encontrado la explicación, y que ésta podrá encontrarse en algún momento.

Es importante aclarar que, durante mucho tiempo, el trabajo que se ha desarrollado con las personas con discapacidad ha tenido su base en la enfermedad, pues eran los médicos y los especialistas de la salud, quienes primero emitian su criterio respecto a qué condición intervenir. A menudo, lo único válido era un certificado de salud donde se describía (sí así se puede llamar) la condición de la persona y su estado físico, y emocional. Es así como los criterios importantes eran los propuestos por los médicos, psiquiatras y psicólogos, quienes con un certificado podían emitir un juicio respecto al estado de una persona.
Por lo tanto, los servicios que se generaban en las diferentes entidades del gobierno o privadas eran dirigidos a una persona con una enfermedad. En verdad ésta ha sido una de las mayores dificultades con las que se ha encontrado la persona con discapacidad al enfrentarse a la sociedad.

Producto de esta situación es que, en el transcurso de las últimas décadas, ha sido necesario tener una nueva visión respecto de lo que significa realmente un diagnóstico de discapacidad o una condición discapacitante. Se ha hecho necesario cambiar el paradigma de atención y de asignación de una condición a una persona, de tal manera que no se haga una clasificación de ella, sino que, más bien, se dé una caracterización de una persona con una condición específica, por esto, en los últimos años se habla de personas con discapacidad, así, se hace referencia a una persona con autismo, a una persona con deficiencia visual, a una persona con retraso mental, entre otros.

Según las clasificaciones que se expandian posteriormente se pone énfasis en la discapacidad y no en la persona, ni el entorno, por esta razón, muchas de las personas que desarrollan diferentes experiencias de trabajo con personas con discapacidad, no están de acuerdo con los códigos que se les asignan a las personas con alguna condición discapacitante, más bien quisieran no dar crédito a la etiqueta y referirse a la persona. Esta ha sido una discusión de muchas décadas y todavía no ha podido ser solucionada, pues la etiqueta tiene mucho significado en diferentes situaciones, aún cuando muchas personas las denominan como término que hace que se dé la segregación.

El objetivo de este artículo es brindar una serie de elementos que permitan el desarrollo de procesos más relacionados con la persona y el entorno, aún cuando en el medio sociocultural en que comparten personas con discapacidad o sin ella, siguen haciendo uso de terminología específica para referirse a ellas 


\section{Los conceptos médicos, limitaciones y rehabilitación}

Como se puede observar, el modelo médico era y sigue siendo muy importante desde mucho tiempo atrás, pues eran los únicos, junto con los médicos y psiquiatras, que desarrollaban listas de clasificaciones diagnósticas, las cuales eran importantes para poder tener medios diagnósticos y de comunicación profesional. Esta condición todavía existe, al darse los documentos denominados:

a. IC-10, y sus versiones anteriores, que es una clasificación internacional de las enfermedades, de mayor uso en Europa y de uso por psiquiátras en Costa Rica.

b. ICE, clasificación internacional de las enfermedades, el cual se ha tomado como base para la clasificación internacional de la discapacidad.

c. Manual Merck, documento de carácter médico, en el cual se hace una clasificación y una descripción médica de las diferentes enfermedades y patologías.

d. DSM-IV, (Manual Diagnóstico y Estadístico de los Trastornos Mentales) y sus versiones anteriores, de la Asociación Americana de Psiquiatría, donde se hace una clasificación y descripción de las diferentes patologías mentales que se presentan en personas, con sus respectivos criterios diagnósticos.

e. Clasificación Internacional de Deficiencias, Discapacidades y Minusvalías -Manual de clasificación de las consecuencias de la enfermedad-, (CIDDM) editado por la Organización Mundial de la Salud (OMS) en 1980, y por el Ministerio de Trabajo y Asuntos Sociales (Instituto de Migraciones y Servicios Sociales de España, IMSERSO) en 1997.

La perspectiva ha ido cambiando en función de la denominación de las alteraciones del desarrollo de las personas, no así en los criterios diagnósticos, por lo tanto la labor de concienciar a la población en temas sobre discapacidad les corresponde a las personas que tienen relación directa o indirectamente con las personas con discapacidad y a las personas con discapacidad.

Por lo tanto, el uso adecuado de términos favorecerá un proceso de comunicación entre la población y se empezará a utilizar un mismo código de comunicación, tomando en cuenta que el respeto a la condición e ideas de los demás, merece el mismo respeto en tanto, la posición individual de cada uno sea respetada, todo partiendo de la idea de los principios de solidaridad e igualdad que justifican las relaciones de las personas.

De esta manera, la tarea de las personas que intentamos accesar el campo de la discapacidad, y sus relaciones con el entorno, está determinada por los procesos de comunicación que se desarrollan con los demás, y de alguna manera, debemos tomar parte en la información y formación de las personas, para de esta manera, abreviar los ruidos en la comunicación.

Tomando en cuenta estos aspectos, se hace necesario que los términos utilizados en las conversaciones sobre personas con discapacidad sean compartidos por todas las personas involucradas en el proceso de comunicación, por esta razón se desarrollan los siguientes apartados:

1. Cuando se habla de medicina fisica, se hace referencia a aquella parte de la medicina que por un lado, se encarga de desarrollar programas de prevención en cualquiera de los tres niveles conocidos, de tal manera que las minusvalías se presenten con menor compromiso, por otro lado, detecta, diagnostica y evalúa todos aquellos procesos que producen una condición discapacitante. También es la encargada de prescribir, dirigir, evaluar y controlar los tratamientos que permiten una mejor calidad de vida a las personas con alguna condición o cuadro discapacitante.

2. Cuando se habla de las limitaciones, este concepto hace referencia a alguna de las siguientes categorías: 
2.1. Deficiencia; cuando las personas por algunas circunstancias específicas pierden una estructura o función fisiológica, psicológica o anatómica. Como se puede ver, este concepto es mucho más amplio que el de trastorno, pues en el concepto de deficiencia se incluye también las pérdidas que las personas puedan tener, por ejemplo, cuando una persona pierde una mano, se convierte en una deficiencia pero no necesariamente en un trastorno.

De esta manera, esta categoría se refiere a la apariencia corporal o a la estructura de un órgano o sistema, y a su funcionamiento, que tiene una causa, así, la persona presenta una situación intrinseca, que puede ser una enfermedad o un accidente, pueden ser de carácter congénito, genético o adquiridas, que le causa esta condición a la persona, y que se ve representada en un aspecto exteriorizado, por la falta de un órgano o un mal funcionamiento de un sistema. Hay que tomar en cuenta que la deficiencia se caracteriza por pérdidas o anormalidades que pueden ser de carácter permanente o temporales.

2.2. Discapacidad, el concepto hace referencia a las restricciones o pérdidas en la habilidad para desarrollar una acción por parte de las personas que padecen una deficiencia, siempre comparando estas acciones con el desempeño de sus coetáneos y personas de su grupo social y de condiciones similares. De esta manera, se hace referencia a las actividades complejas e integradas que se espera que realice una persona, o de un cuerpo que funciona como un conjunto de órganos y sistemas en una unidad, entre ellas se pueden mencionar las diferentes conductas y tareas que lleva a cabo una per- sona, entre ellas trasladarse, hablar, caminar, dibujar, entre otras.

Como puede determinarse del concepto, la discapacidad hace referencia más bien a la desviación de una norma, en cuanto a la actuación que debe tener una persona, y no hace referencia como la deficiencia a la función de un órgano o sistema del cuerpo.

Así, este concepto se ve caracterizado por los excesos o defectos en relación con la actividad de las personas o con su conducta, estos pueden ser de carácter temporal o permanente, también pueden ser reversibles o irreversibles, y progresivos o regresivos. En este sentido, la discapacidad se caracteriza por la objetivación que hace la persona de su condición, así, la limitación funcional se plasma o manifiesta como algo real en la vida diaria, y que influye por lo tanto, en las actividades que realiza una persona en su entorno, y en los diferentes niveles del mismo. De esta manera, la discapacidad se hace más evidente en tanto la persona toma conciencia de un cambio en su identidad, y además afecta los diferentes aspectos de la conducta del ser humano, a saber; lo físico, lo psicológico y lo social, con la dificultad de no poder determinar claramente los aspectos de carácter médico o sociales de la actividad.

Es importante tener claro que la categoría de la discapacidad hace especial referencia al concepto del accionar de la persona, a las actividades que ella realiza, a lo que ocurre en la vida práctica de la persona, y quizás acepta muchos matices de la conducta de la persona, o sea ser parte en la mayoría de los casos del potencial de la persona, y no en la emisión de valor de un juicio de carácter negativo o categórico, lo cual sería de alguna manera ofensivo y estigmatizante, situación que no se quiere llevar a cabo dentro de la perspectiva de persona y entorno.

2.3. Minusvalia (Desventaja), el concepto hace referencia a la limitación que le impide a una persona el buen desarrollo de sus funciones, teniendo en cuenta la edad, 
su género y las características socioculturales que se dan en el entorno en el cual se desarrolla la persona.

Se hace referencia a una situación de desventaja para una persona, que puede estar determinado por una deficiencia y/o una discapacidad determinada. Es la valoración social del accionar de las personas en función de lo que ha sido determinado como norma en la sociedad.

Así, es posible que la valoración sea dada por la persona o por parte del grupo de semejantes con quienes comparte en el entorno.

Por otro lado, el accionar de la persona puede ser valorado bajo las reglas que se establecen culturalmente para un grupo específico, así, es posible que la persona se halle en situación de desventaja en un grupo, o situación pero no en otros grupos o situaciones, todo dependerá del momento, el espacio, la condición y el rol que le corresponde en el grupo en el que actúa.

Y también, el juicio de valor de desventaja que se emite siempre es dado con respecto a la persona que porta alguna condición discapacitante, o que está afectada.

Como se puede ver la situación de desventaja, debe medirse en función de las demás personas, por lo tanto, se deben tener muy claro los valores sociales del grupo en el cual se desarrolla la persona, pues los valores también se ven influenciados por la forma en que está organizada institucionalmente una sociedad. De esta manera, la forma en que las personas que se encuentran en situaciones de desventaja actúan, tiene mucha importancia en el desarrollo del concepto de sí mismo y la delimitación de sus potenciales y sus debilidades, quienes pueden encontrarse con muchos problemas para determinar o modificar su realidad, y que por lo tanto, la sociedad va a responder de diferente manera a las deficiencias y situaciones de desventaja, pues todo dependerá de la visibilidad y el grado de ellas.

De alguna manera, la situación desventajosa o minusvalía se ve caracterizada por una discordancia entre la actuación o estado de la persona y las expectativas que se tienen de grupo, principalmente con el que ella interactúa. De alguna manera la resistencia al cambio y las dificultades de participación de la persona en las actividades del entorno, acrecientan la situación de desventaja, por lo tanto, se puede decir que la minusvalía o desventaja es de carácter social, y que se da, en tanto representa las consecuencias sociales y del entorno que dificultan la participación en igualdad de condiciones, por la presencia en las personas de deficiencias y discapacidades.

La forma en que se han presentado las categorías dan la idea de ser una función lineal, sin embargo, cada una de ellas pueden darse en forma separada o integrada o relacionada, todo va a depender de las condiciones en que se presente la condición discapacitante, de las características de la persona, de la forma en que el entorno valora el actuar de las personas y como en general, un grupo social tiene determinada su organización y el papel que cada miembro desempeña en él.

Como se puede observar, las clasificaciones o agrupaciones de las condiciones se dan más que todo en función de una mejor aproximación a su estudio, es la forma en que las personas pueden llegar a una mayor comprensión de los conceptos que se utilizan en el ámbito de la discapacidad. La idea es evitar que todas las personas sean ubicadas en un solo rango, y que todo lo que a ellas se refiera en función de su condición sea valorado desde una única perspectiva, sino que más bien se pueda hacer valer un abanico de posibilidades que permita un mejor acceso de la persona a las diferentes actividades del entorno en el que se desarrolla y que eso le permita una mejor calidad de vida, tanto a la persona como a las personas que con ellas comparten un espacio y momento, en el continuum del desarrollo. Es hacer valer a la persona y no su condición, es brindar la mejor oferta en todas las áreas del desarrollo, es la búsqueda del ser un miembro de la sociedad y no un miembro más.

Las categorías de las limitaciones pueden verse representadas de la siguiente forma: 


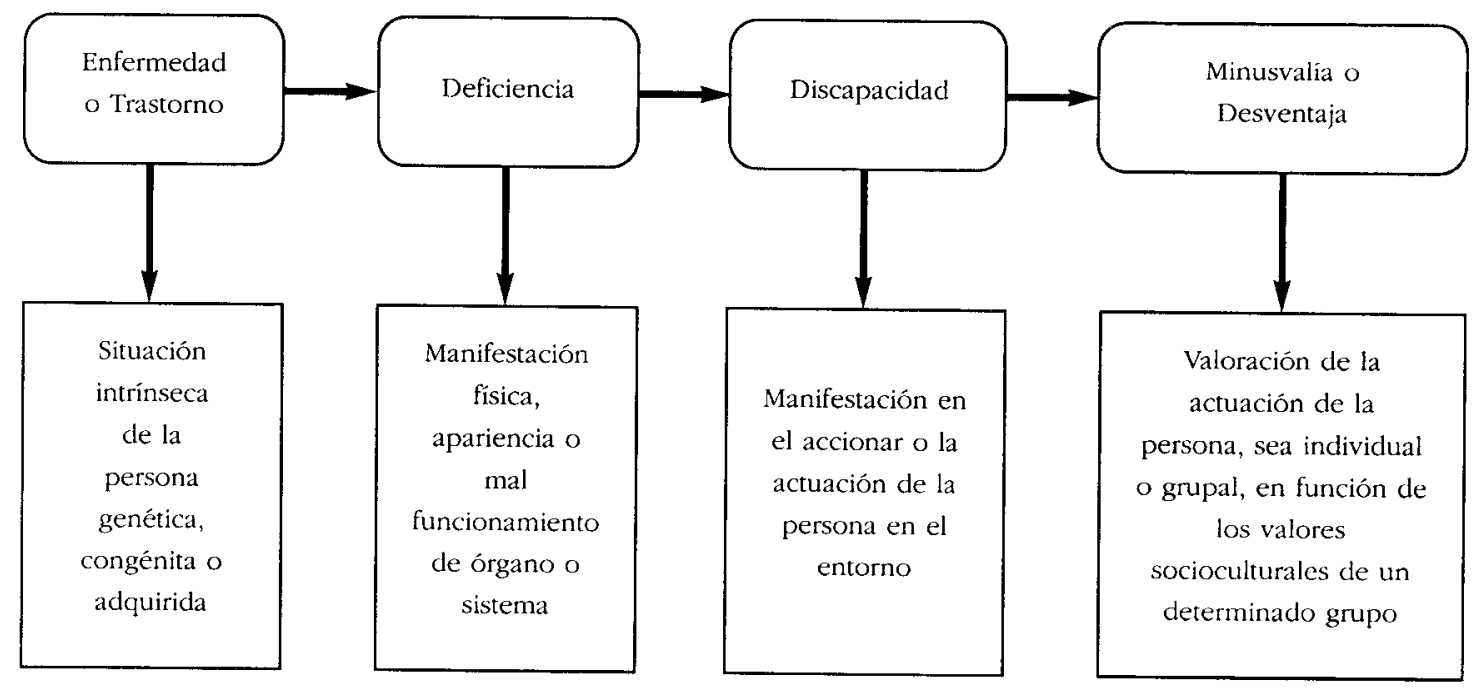

Esta presentación de las categorías de alguna manera favorece el hecho de que puede ser un modelo que se tome en cuenta para dar la mejor respuesta a las necesidades de las personas y de realizar una mejor valoración de las situaciones que en lo que respecta a la intervención y propuestas de atención por parte de los diferentes profesionales que tienen relación con las personas con alguna condición discapacitante.

No obstante, no pretende ser de ninguna manera una única forma de percibir las condiciones limitantes por las que atraviesa una persona, sean estas de carácter temporal o permanente, ni tampoco se pretende que todo encaje dentro del modelo planteado. Es importante, que el profesional que haga uso de este modelo comprenda que cada situación es particularmente diferente y que por lo tanto, se debe buscar la mejor opción, dependiendo de la persona, su edad, su género, su condición tanto física, como socioemocional, cada persona debe ser tratada desde sus propias características, y ser vistas como un ser biopsicosocial, que presenta sus fortalezas y debilidades.

También se debe tener en cuenta la temporalidad de la situación que atraviesa la persona, para así, no hacer de la caracterización de los servicios una eterna respuesta asistencial o crear las dependencias de las personas hacia los servicios. La respuesta debe darse en función de los siguientes aspectos: grado, temporalidad, accesibilidad, edad y condición sociocultural; sin embrago, el proceso debe ser evaluado mediante el seguimiento del mismo, de tal manera que cada vez todo se adecúe más a las condiciones temporales de la persona, las cuales pueden variar de tiempo en tiempo.

3. Otro concepto importante a revisar es el de Rebabilitación, que consiste en el proceso que prescribe, dirige, supervisa y evalúa los programas que se desarrollan en la adaptación y la readaptación de las personas con alguna limitación, de tal forma que se puedan restaurar el movimiento, las funciones de comunicación y la eficiencia psicológica, social y vocacional, dependiendo de las caracteristicas y necesidades de cada persona, por medio de los recursos médicos, familiares y comunitarios.

Se pude decir que los programas de rehabilitación pretenden que se disminuya el impacto de la limitación de tal manera que se ofrezca una mejor oferta de coberturas y de desarrollar procesos de participación de la 
persona con alguna condición discapacitante. De alguna manera tiene características importantes, entre ellas, es un servicio con carácter local y descentralizado, el cual se integra a un sistema de salud que permita la supervisión y la referencia, por otro lado, debe ser desarro- llado por algún miembro de la comunidad que esté capacitado para desarrollar el proceso de una manera dinámica y funcional. manera:

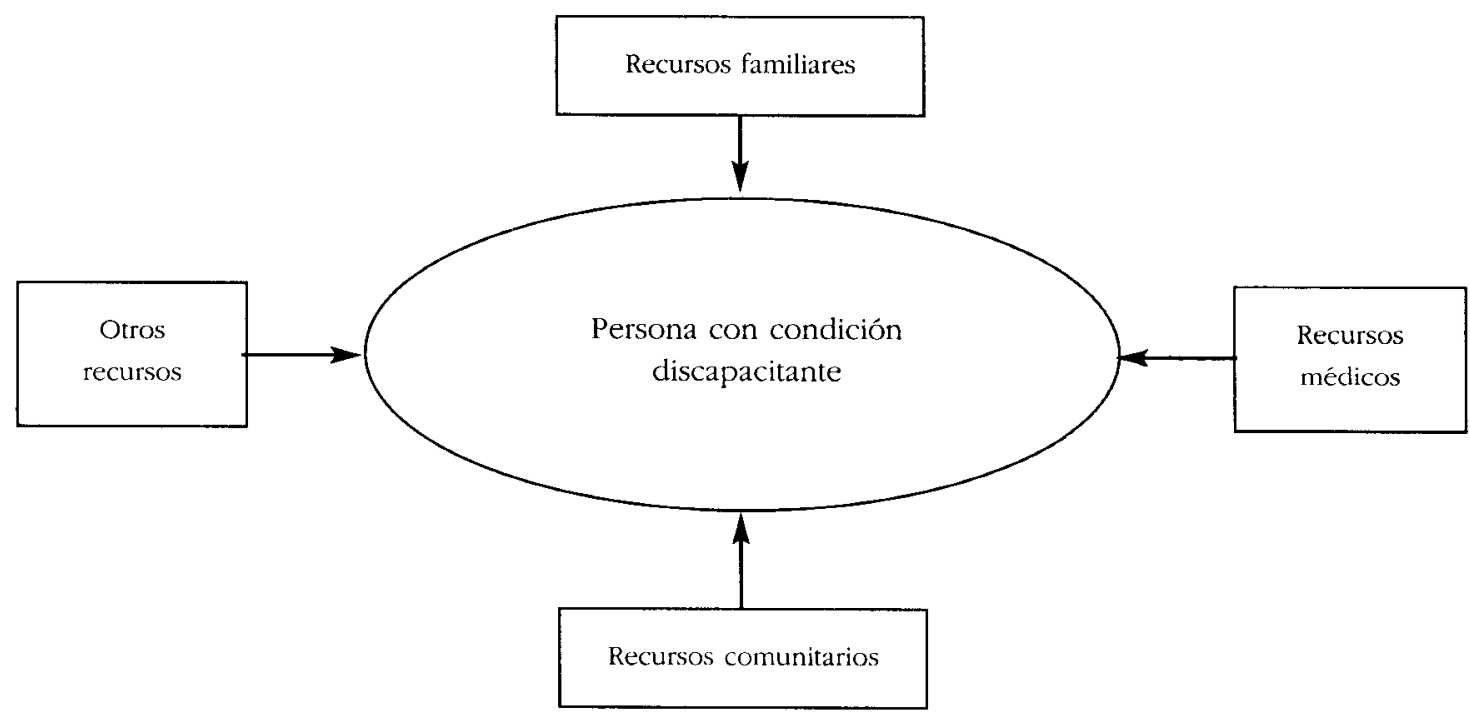

Como se puede ver el enfoque responde al modelo ecológico, el cual presta especial atención tanto a la persona como al entorno, y es así, como se espera brindar la mejor oferta a las personas con discapacidad y sus familias.

\section{Entre los conceptos que se han desarrollado dentro del nuevo paradigma y se consideran importantes de comentar, se encuentran:}

\subsection{Igualdad de oportunidades:}

Según Asamblea Legislativa, 1996, este concepto hace referencia al

"Principio que reconoce la importancia de las diversas necesidades del individuo, las cuales deben constituir la base de la planificación de la sociedad con el fin de ase- gurar el empleo de los recursos para garantizar que las personas disfruten de iguales oportunidades de acceso y participación en idénticas circunstancias" (p. 8).

En este sentido se puede ver cómo el principio que se define en el párrafo anterior, determina que todas las personas sin importar su condición, raza o credo deben tener la oportunidad de participar en igualdad de condiciones que todas las personas, y por lo tanto, las personas con discapacidad o sus encargados deben hacer que se cumpla el principio. El cumplimiento de este principio hace que la persona con discapacidad participe en su entorno, igual que lo hace cualquier persona con o sin discapacidad.

\subsection{Equiparación de oportunidades:}

Según la Ley 7600, "Ley de Igualdad de Oportunidades para las personas con discapacidad" del 29 de mayo de 1996, el concepto hace referencia al 
"Proceso de ajuste del entorno, los servicios, las actividades, la información, la documentación así como las actitudes a las necesidades de las personas, en particular de las discapacitadas." (Asamblea Legislativa, 1996, p. 9).

En lo que se refiere a este concepto, es importante aclarar que hace una mayor referencia a que el entorno permita que la persona con discapacidad se desarrolle con las mismas oportunidades que tiene cualquier persona de su comunidad, y que tenga acceso al igual que todos los miembros de su entorno de poder desarrollarse y favorecerse de los recursos, medios, actividades y procesos de crecimiento personal en que participan que interactúa en el entorno, siempre en la búsqueda de una mejor calidad de vida, tanto para ella como para los miembros de su familia y comunidad.

\section{Consideraciones sobre los conceptos}

El abordaje de la discapacidad ha sido una temática de actualidad que ha permitido el desarrollo de diferentes términos y procesos, de tal manera que se ha trasladado la compresión de los conceptos más a una condición de la persona, y no a calificar a las personas por su condición. La respuesta a las características y necesidades de las personas permite, por lo tanto, dar un papel importante al entorno donde convive la persona, de esta manera, la labor que el entorno debe cumplir es favorecer los procesos de participación de las personas con discapacidad en igualdad de condiciones y oportunidades, a los miembros de su familia y comunidad.

Se le da un mayor respeto a las condiciones y caracteristicas de las personas y se favorece la toma de decisiones por parte de las personas con discapacidad, así, se respeta el hecho de que cada persona pueda expresar sus deseos, necesidades e inquietudes, pero principalmente que puede decidir por sí misma, siempre y cuando su condición lo permita.

La terminología utilizada puede variar en función del uso que en el momento pue- da beneficiar a la persona con servicios y recursos, y de alguna manera, estos téminos permiten una mejor comprensión por parte de toda una comunidad y esto favorece la actitud de los demás. Es importante recordar que la terminología está referida a una condición de la persona, pero que los aspectos legales y la terminología pretenden motivar actitudes de comprensión, de solidaridad y sobre todo de respeto a la diferencia del ser humano.

A menudo las personas hacen uso de los términos a su antojo, en función de sus necesidades e intereses, sin embargo, es posible que los téminos cambien, que las personas cambien, y en general que la actitud de las personas cambie, pero, la condición discapacitante y su efecto sobre el desarrollo individual y social de la persona permanecerán en tanto no se respete la diferencia entre las personas, y se permita a todos participar en igualdad de condiciones y con equidad de oportunidades.

Por lo tanto, la tarea de concienciar a las personas con respecto a la utilización adecuada de la teminología en cuanto a la temática de la discapacidad, debe ser abordada desde todos los niveles de la educación costarricense y por todos los docentes y padres de familia, para así construir una sociedad más consciente, solidaria y respetuosa de todos sus miembros.

\section{La participación de la persona}

Todas las personas deben participar en las diferentes actividades y en todos los aspectos de la sociedad, sin importar su condición. A menudo el solo referirse a una persona por alguna de sus características determina el grado de aceptación o de rechazo que se le tiene a ella. Es difícil sin embargo, referirse a una persona sólo por su nombre, pues la mayoría de las veces la sociedad ha asignado a cada uno un estatus que lo identifica, pues se vive en una sociedad donde todo tiene nombre y muchas veces apellido. 
Muchos son los términos que se han utilizado para denominar la participación de las personas con discapacidad en los aspectos sociales, así la integración, la rehabilitación, la normalización, la inclusión entre otros.

No obstante, estas denominaciones se hacen en función de la productividad que pueda tener la persona con discapacidad en la sociedad, "tanto como produces, asi vales", y se ve la integración a la sociedad solamente como el resultado de la valía económica que la persona pueda tener, se dejan de lado otros aspectos más importantes como los valores, las aptitudes, las destrezas, la capacidad de tomar sus propias decisiones y otras características de convivencia necesarias para todas las personas.

Hace muchos años, las etiquetas y las clasificaciones de la discapacidad no tenían tanta importancia como en la actualidad. En aquellos años lo importante era buscar la forma en que las personas con discapacidad pudieran sobrevivir. Muchas de estas personas morían, pues no contaban con las capacidades que les permitieran desarrollar las actividades de supervivencia necesaria en el grupo social en que vivían, muchas de estas personas perecian a manos de sus familiares o de profesionales y muchos otros eran abandonados a su suerte.

Posteriormente, estas personas con discapacidad fueron producto de la burla de las personas sin discapacidad y muchos otros eran usados en espectáculos para provocar en las personas reacciones de rechazo o de lástima, principalmente aquellas personas con deformidades físicas. Todas estas personas fueron víctimas de un proceso de exclusión de aquellas actividades que mejoraban la vida de los habitantes de una región. Estas personas no tenían derecho a nada.

De alguna manera, estas situaciones de abandono producen en las personas sin discapacidad la idea de mejorar la condición de aquellas personas con alguna discapacidad, sin embargo, la respuesta siempre viene dada por un grupo de personas sin discapacidad, muchas veces sin tomar en cuenta las ideas o necesidades de las personas con discapacidad. A partir de estas ideas nace entonces la necesidad de usar etiquetas y sistemas de clasificación, sin embargo muchos consideran que "tienen la función de excluirlos de la sociedad" (Heward, 1998, p. 9), mientras que otros consideran que una buena clasificación permitiría "proporcionarles los diferentes servicios que requieren las personas para poder permitirles una mejor participación en el entorno en que se desarrollan. Heward, 1998, comenta "Una taxonomía, además de tener un interés científico, legal y educativo, incluye consideraciones de tipo ideológico, político y ético". (p. 10).

Muchos pueden ser los beneficios que pueda tener el uso de la etiqueta, entre ellos se podrían mencionar: las respuestas de tratamiento a personas con una condición discapacitante están relacionadas con las categorias que se han desarrollado, muchas veces cuando la conducta de las personas con discapacidad no común a la de una persona con discapacidad, la comprensión de las personas puede ser mayor, la comunicación que se establece entre los profesionales miembros de un equipo, se puede ver favorecida al poder desarrollar investigaciones tendientes a mejorar la condición de las personas con discapacidad, por otro lado los aspectos legales pueden ajustarse más a la realidad de las personas con discapacidad y además, el desarrollo de programas que mejoren la calidad de vida de las personas muchas veces requieren de financiamiento, que se consigue mediante apoyos a grupos de personas con discapacidad.

No obstante, existen también algunas desventajas en el uso de etiquetas. Entre ellas se puede mencionar, que la clasificación o el uso de etiquetas hace más referencia a la discapacidad de la persona y no a sus destrezas y habilidades, muchas veces cuando se comenta la condición de una persona las expectativas de los padres de familia y profesionales pueden verse disminuidas, pues no la consideran capaz de realizar muchas cosas y, por lo tanto, le exigen lo mínimo. Por otro lado, los profesionales y los padres de familia 
acostumbran justificar la conducta de las personas con discapacidad, haciendo alusión a la discapacidad, por otro lado, centran la problemática en la persona y no en los aspectos sociales y/o instruccionales que la afectan. Muchas de las personas que han sido etiquetadas por alguna razón, tienen muy baja autoestima, y pueden ver afectadas las relaciones con los demás compañeros, quienes tienden a mofarse de ellas y a ridiculizarlas o rechazarlas. El mayor problema del etiquetado es que parece un tatuaje que se le pone a la persona, una vez que se le pone, no se le quita o es muy dificil eliminarlo. Por otro lado, el diagnóstico realizado a una persona con alguna discapacidad, requiere de una gran cantidad de profesionales y recursos económicos y materiales para desarrollar los programas que mejor respondan a sus necesidades.

Sin embargo, el uso de las etiquetas ha sido utilizado para poder organizar la administración de los servicios que se le pueden brindar a la persona en las áreas sociales, educativas y de salud. De esta manera, para que una persona se vea favorecida por un programa específico es necesario que se pueda incluir dentro de las categorías legales prescritas para recibir el servicio que se solicita, si no cumple con estos requisitos es muy posible que no reciba el servicio.

No obstante, aunque la identificación y la clasificación de las personas con discapacidad parece ser necesaria, la mayoría de las personas no están de acuerdo con ellas, y pocos se muestran satisfechos, pues a menudo los criterios diagnósticos exteriorizados por los profesionales, son poco eficaces. Por esta razón, muchos otros consideran que se hacen diagnósticos sin sentido y esto limita en mucho, el diseño y planificación de los servicios de apoyo que aquella persona puede requerir para mejorar su calidad de vida.

\section{De la rehabilitación a la integración}

Producto de todo el análisis que se ha desarrollado en los últimos años con respec- to a la persona con discapacidad, se han generado una serie de servicios y apoyos que van desde su atención en el campo de la salud en el área de la prevención, hasta lo denominando inserción laboral. Es así como en los últimos años se han organizado servicios que presten a las personas con discapacidad un apoyo desde el punto de vista de sus necesidades específicas.

La rehabilitación pasa de ser un concepto basado en términos médicos a una posición más clara y mejor definida, concebida así, como una respuesta a los asuntos morales que se asocian con la relación existente entre una persona en particular y la sociedad en que vive (Seyfried, 1996), por lo tanto, las personas con discapacidad y la sociedad deben sufrir una serie de cambios, de tal manera que la persona con discapacidad participe en igualdad de oportunidades y condiciones en el grupo social, o sea, que sea visto como un miembro más de la comunidad.

De esta manera, la sociedad debe encargarse de desarrollar un cambio de actitud hacia la persona que de alguna manera era partícipe en un proceso de exclusión, sin embargo, esta tarea se ha visto opacada, o la visión se ha perdido, y las personas con discapacidad aún con todos los derechos actuales siguen siendo de alguna manera excluidos, como la gran mayoría de los grupos minoritarios.

Esta pérdida de la visión de la rehabilitación se debe a que la rehabilitación ha sido tomada como una colección de técnicas y medidas compensatorias que se aplican a una persona con discapacidad (más bien con minusvalia), y se pierden de vista los cambios que deben darse en la sociedad (Seyfried, 1996, Obando, 1990), de esta manera lo que se denominaría un acto social, pasa a ser un proceso orientado a las personas con discapacidad, como herramienta que le sirva para su transformación y adaptación a ciertos círculos sociales.

La rehabilitación debería permitirle a la persona con discapacidad la adquisición de habilidades específicas, y según comenta Seyfried, 1996 debería ser un "proceso en el 
cual las personas con minusvalía son capaces de incrementar su independencia y autonomía por medio del desarrollo de sus habilidades" (p. 54). En este sentido debe darse un proceso de educación escolar, de formación profesional, el entrenamiento en habilidades, pero no detenerse solamente en estos aspectos, sino más favorecer los procesos de autonomía y desarrollo personal.

De esta manera, la rehabilitación permitiría pasar con más facilidad a un proceso de integración de la persona con discapacidad en la sociedad, o sea la participación de la persona en igualdad de condiciones y oportunidades.

Tomando en cuenta el concepto anterior, la integración debe verse como la "capacidad que tiene la sociedad para cambiar de tal forma que la persona con desventaja participe con iguales derechos y en igualdad de oportunidades" (Seyfried, 1996, p. 54). Así, la rehabilitación sería todo un éxito, pues la persona con desventaja debe ser capaz de competir con personas sin discapacidad, pero esto requiere de la participación de las personas, en este sentido, la integración se debe dirigir más al medio y a la sociedad, de alguna manera que se pueda ver el cambio en las circunstancias de la vida y la adaptación a las necesidades de las personas con alguna condición discapacitante, de esta manera lo que se pretende es hacer más cordiales y solidarias las relaciones entre las personas, que se relacionan o intervienen en el contexto como una comunidad.

Si se toman en cuenta las ideas anteriores, entonces la integración más que un proceso es un conjunto de relaciones de igualdad que se dan entre las personas, pero no puede haber subordinación de ninguna de ellas, así, se transformarán las relaciones entre las personas con y sin discapacidad, en igualdad de condiciones y conscientes de que se reconocen la diversidad de cada una de ellas, pero que estas diferencias lo único que permiten es el respeto a las características de cada una como elemento complementario para desarrollar actividades que favorezcan la convivencia del grupo social.
En resumen, la rehabilitación tiene sentido en tanto se da la integración, de esta manera, el éxito de la integración estaría basado en la actitud que la sociedad tenga hacia las personas con discapacidad y la receptividad hacia la idea de que las personas con desventajas deben participar en igualdad de oportunidad que el resto de la comunidad y que por lo tanto no deben ser objeto de discriminación, marginación o segregación.

La rehabilitación es entonces un proceso en el que la sociedad debe brindar apoyo y acompañar a la persona con discapacidad, de tal forma que el entorno se transforme y adapte a las exigencias de la persona. De esta manera, se desarrollan la desinstitucionalización y la normalización, como procesos contrarios a la segregación, o formas de exclusión social de las personas con discapacidad unido a la ubicación en un lugar determinado para las personas. Por otro lado, la normalización sería vista como las formas de comunicación y cooperación que desarrolla la sociedad entre las personas con discapacidad y sin discapacidad, esto siempre tomando en cuenta el entorno de la persona, o sea compartir en los ambientes naturales de las personas, así la persona tiene derecho a participar en actividades de la vida, tanto en el campo familiar como en el laboral, de ocio y de recreación, estas condiciones le darían un mejor desarrollo de su estatus social debido al desarrollo de habilidades $\mathrm{v}$ competencias, o sea, la rehabilitación toma en cuenta tanto a la persona con discapacidad como el sistema social de grupo al que ella pertenece.

No obstante, los procesos de desinstitucionalización deben contar no solo con la eliminación de las instituciones, sino también involucrar aspectos relacionados con los cambios permanentes que se producen en las estructuras, el contenido y organización de las instituciones sociales, lo mismo que la forma en que trabajan los empleados y como se confeccionan las estrategias de trabajo para satisfacer las necesidades, intereses, requisitos y perspectivas de las personas con discapacidad. 
Otro aspecto importante a tomar en cuenta es el concepto de aceptación de la diferencia, este implica delimitar entre tú y yo, de alguna manera es participar en relaciones de igualdad entre las personas con y sin discapacidad, pero que ambos tienen necesidades e intereses diferentes a los propios, es un proceso de desarrollo de tolerancia, de respeto a la individualidad, es ser con cada una de las personas, sin dejar de ser en cada uno de nosotros, es de alguna manera compartir con el otro en su calidad de diferente.

El resultado del proceso anterior, implica por lo tanto, participar en procesos de comunicación y cooperación que eviten la exclusión de las personas, sin importar su condición. Es así como el entorno y la persona adquieren un papel relevante en el proceso, es un continuo en las relaciones que favorece la solidaridad y el desarrollo de las personas, en el respeto a la diferencia.

\section{Los conceptos de limitación}

Como se habrá podido deducir de los apartados anteriores, los procesos de desinstitucionalización no aseguran de ninguna manera la participación de las personas en igualdad de oportunidad, ni en la vida diaria, ni en el aspecto laboral, ni en el ocio y la recreación. Para esto sería necesario según Seyfried, 1996 que la discapacidad se concibiera como parte integral de la normalidad, sin embargo las definiciones actuales no cumplen con este requisito.

Así, los conceptos discutidos en el artículo anterior, no caen dentro de este rango, pues todas ellas están basadas en el concepto de normalidad de la Organización Mundial de la Salud (Heward, 1998; Seyfried, 1998; Ministerio de Trabajo y Asuntos Sociales, 1997, Revista Minusval, 1984). Un concepto que podría responder más a los cuestionamientos de las personas con discapacidad y aquellas que trabajan con personas con discapacidad debería estar basado en los Derechos Humanos, donde se vea a las personas en desventaja con los mismos derechos, las mismas oportunidades y opciones de las demás personas. No obstante, las definiciones actuales basan su justificación en aspectos médicos, en la imposibilidad de hacer cosas, en fin, se ve a la persona como alguien incapaz de realizar alguna tarea.

Seyfried, 1998, menciona a Momm, quien propone una definición positiva de la siguiente manera, "todos aquellos que a pesar de sus numerosas habilidades experimentan dificultades significativas y desventajas en su vida con base a la incapacidad de la sociedad o su medio para aceptar su diferencia determinada por su discapacidad" (p. 60). De esta manera, la sociedad incluyéndonos a todos, es la responsable de la exclusión y la integración de las personas con discapacidad en la sociedad.

Así, las personas podrán desempeñar su trabajo bajo sus condiciones y no podrá ser segregado por las ideas de los empleadores, participaría en igualdad de condiciones, el estado debe brindar los servicios sociales y los apoyos necesarios a las personas con discapacidad, también es necesario que la sociedad tome en cuenta dentro del concepto de normalidad, los aspectos relacionados con la discapacidad, para que de esta manera pueda crear un entorno que brinde un mayor apoyo a las personas con discapacidad y minimice de esta manera, los efectos de la discapacidad.

Es por esto, que la sociedad debe darse a la tarea de buscar la cooperación con base a la igualdad y la aceptación de la diferencia, como una experiencia enriquecedora para todos, donde cada persona aporta en relación con sus características, habilidades y potencialidades dentro de un trabajo de cooperación entre los participantes. Es crecer desde aportes heterogéneos de las personas, donde la dinámica de ser diferente es lo común.

De alguna manera, la rehabilitación debe ser un trabajo que no solamente compete a los profesionales sino más bien, a las personas con discapacidad, sin embargo, es necesario dar una relación muy estrecha en 
todos aquellos programas que se desarrollen como respuesta a las exigencias de las personas con discapacidad, o sea todas las decisiones que se tomen en cuanto a su rehabilitación deben ser compartidas y discutidas con las personas a las que se dirige el apoyo. La idea de este concepto es por lo tanto, el desarrollo de los procesos de autoayuda y responsabilidad de las personas, o sea deben ser corresponsables del proceso. No es solo responsabilidad del estado brindar los medios y recursos económicos y humanos, es más bien una tarea compartida donde todos apoyan desde diferentes esferas.

Sin embargo, todo programa que se quiera desarrollar debe contar con muy buena estructura organizativa, contar con personal cualificado y que se haga uso de métodos de trabajo adecuados a las necesidades e intereses de las personas con discapacidad a las que va dirigido. De esta manera se brindarán los apoyos necesarios para un buen servicio de rehabilitación.

Se debe pasar de un modelo donde se desarrollen programas de rehabilitación basados en la comunidad, a programas de rehabilitación desarrollados con la comunidad, o sea, todos los miembros son corresponsables de los programas que se desarrollan en beneficio de todos. La actitud de la comunidad es muy importante, y esto se logra de una mejor manera, si se sienten parte del proceso, y no simples observadores, es aquí donde está la tarea de muchas personas que desarrollan estos programas, en motivar a todos a trabajar por un bien común, la participación en igualdad de oportunidades y en equiparación de oportunidades. Es ver a la persona como un miembro más de la comunidad, es ver a la persona y no a la condición, es aceptar que todos tienen derecho a compartir un espacio, y convivir de la mejor manera posible.

Se debe recordar, que todos los programas que se implementen deben desarrollarse en situaciones naturales de las personas, así, en su hogar, en la escuela, en su lugar de trabajo, en su comunidad, y se debe respetar la autodeterminación de la elección del servicio, sin necesidad de ser censurado.
Para resumir, los programas de rehabilitación y participación deben ser la respuesta a todas aquellas personas que los requieran como una forma de desarrollarse como seres humanos con la mejor calidad de vida, y tomando en cuenta sus propias características, es el proceso mediante el cual, el estado o la comunidad organiza o define un marco de acción, tomando en cuenta las características y necesidades de las personas y evaluando el entorno en que la persona vive, con la intención de desarrollar un programa en el ambiente natural, y organizando los servicios y apoyos que se le pueden brindar a las personas y seleccionando la mejor metodología de trabajc.

Todas las comunidades son responsables de sus miembros, y por lo tanto, deben brindarles las mejores posibilidades de desarrollo y participación en los entornos menos restrictivos, es permitirles ser a todos en la medida de las posibilidades, pero evitando los obstáculos que pudiesen detener el proceso o alterar el curso del desarrollo de las personas con discapacidad. Es una tarea de todos en conjunto.

\section{Consideraciones finales}

La situación actual con respecto a las etiquetas, es muy común entre las personas con discapacidad o sin ella sin embargo, se ha visto que muchos de los programas que se han desarrollado van dirigidos a la situación que presentan las personas que son ubicadas dentro de un grupo determinado. Por lo tanto, se considera que esta será una discusión que facilitará una mejor comprensión hacia el enfoque centrado en la persona.

La denominación que se haga de un grupo con una condición específica, no cobra importancia sino hasta que se den situaciones de rechazo, marginación y segregación, todos estos aspectos están relacionados con la actitud de la sociedad ante la discapacidad y hacia las personas con discapacidad. Todos debemos colaborar en este aspecto, e 
ir desarrollando estrategias que permitan una mejor comprensión de las personas con discapacidad y su situación.

Lo importante es tomar parte en el desarrollo de programas de rehabilitación y de participación de las personas en las diferentes comunidades, pero que los programas no se basen en la comunidad, sino que más bien sean desarrollados en ellas, sin cambiar el entorno natural de la persona, es participar del proceso, siendo parte de la comunidad, con igualdad de deberes y derechos.

El estado como ente regulador de un servicio social, debe contar con programas que respondan a las necesidades, características e intereses de las personas con discapacidad, que hayan sido formulados no solo por profesionales, sino por todo un equipo que incorpore a la persona con discapacidad, a personal calificado, a los servicios comunitarios y todos aquellos apoyos con que se cuenta para el logro de los objetivos de los programas de rehabilitación, que son la independencia, la autonomía y el desarrollo de habilidades que le permitan una mejor participación en la comunidad a las personas con discapacidad.

Los proceso de rehabilitación por lo tanto, tienen una responsabilidad compartida tanto por las personas con discapacidad y sus familias, como por el estado y las comunidades, por lo tanto, la respuesta debe darse en forma integrada, y no sobrecargando a uno de los miembros que se consideran responsables de la acción.

El trabajo de todos los profesionales en el área de la discapacidad es por lo tanto, una acción que se debe ver reflejada en un ajuste social al concepto de la discapacidad y al cambio de actitud hacia las personas con discapacidad, de tal manera, que se les brinde el lugar justo que se merecen como miembros de una comunidad.

\section{Referencias bibliogrāficas}

Asamblea Legislativa. Ley 7600. Igualdad de oportunidades para las personas con discapacidad. San José: Imprenta Nacional. 1996.

Enfermedad, Deficiencia, discapacidad y minusvalía. En: Revista Minusval. № 46. Dic. 1984. Pp. 9-12.

Heward, W. Niños excepcionales: Una introducción a la educación especial. Quinta Edición. España: Prentice Hall. 1998.

Hurlock, E. Desarrollo del niño. 2da. Edición. México: McGraw Hill. 1990.

Inserso "Enfermedad, deficiencia, discapacidad y minusvalía" En: Revista Minusval. № 46. Dic. 1984. Pp. 9-12.

Ministerio de Trabajo y Asuntos Sociales Clasificación Internacional de Deficiencias, Discapacidades y Minusvalías. 3 Edición. Madrid: Instituto de Migraciones y Servicios Sociales. 1997.

Ministerio de Trabajo y Asuntos Sociales. La discapacidad en el Siglo XXI. Plan de Acción. Una propuesta de futuro. Madrid: Instituto de Migraciones y Servicios Sociales. 1997.

Ministerio de Trabajo y Asuntos Sociales. La discapacidad en el Siglo XXI. Plan de Acción. Una propuesta de futuro. Madrid: Instituto de Migraciones y Servicios Sociales. 1997.

Obando, O. F. Rehabilitación Profesional: Fundamentación, Evolución y Acciones Interdisciplinarias. San José, Costa Rica: EUNED. 1990.

Obando, O. F. Rebabilitación Profesional: Fundamentación, Evolución y Acciones Interdisciplinarias. San José, Costa Rica: EUNED. 1990. 
Papalia y Wendkos. Psicologia del Desarrollo. Tercera Edición. México: Editorial Mc Graw Hill. 1998.

Seyfried, E. Políticas de rehabilitación integral. En: Ministerio de Trabajo y Asuntos Sociales. La discapacidad en el Siglo XXI. Plan de Acción. Una propuesta de fuluro. Madrid: Instituto de Migraciones y Servicios Sociales. 1996.

Walker y Shea. Manejo Conductual. Un enfoque práctico para Educadores. México: Editorial Manual Moderno. Traducción de la $3^{\text {a }}$ Edición. 1987. 